\title{
DATA AND PERSPECTIVES
}

\section{Inequality in Human Development across the Globe}

\author{
IÑAKI PERMANYER AND JEROEN SMits id
}

The Human Development Index is the world's most famous indicator of the level of development of societies. A disadvantage of this index is however that only national values are available, whereas within many countries huge subnational variation in development exists. We therefore have developed the Subnational Human Development Index (SHDI), which shows within-country variation in human development across the globe. Covering more than 1,600 regions within 161 countries, the SHDI and its underlying dimension indices provide a 10 times higher resolution picture of human development than previously available. The newly observed within-country variation is particularly strong in low-and middle-developed countries. Education disparities explain most SHDI inequality within low-developed countries, and standard of living differences are most important within the more highly developed ones. Strong convergence forces operating both across and within countries have compensated the inequality enhancing force of population growth. These changes will shape the twenty-first century agenda of scientists and policy-makers concerned with global distributive justice.

\section{Introduction}

Since 1990, the United Nations Development Programme (UNDP) has reported on a yearly basis the values of its flagship indicator: the Human Development Index (HDI; UNDP 1990-2018). This HDI-which indicates countries' combined achievements in education, health, and standard of living-has become over time the key reference indicator to assess countries' socioeconomic performance and is currently employed in Wide

Iñaki Permanyer, Centre d'Estudis Demogràfics (a member of the CERCA Programme / Generalitat de Catalunya), Carrer de Ca n'Altayó, Edifici E-2, Campus de la UAB, Cerdanyola del Vallès, 08193, Spain, Email: ipermanyer@ced.uab.es. Jeroen Smits, Global Data Lab, Institute for Management Research, Radboud University, PO Box 9108, 6500HK, Nijmegen, The Netherlands. Email: j.smits@fm.ru.nl. 
-ranging areas of the social sciences (e.g., Bongaarts and Watkins 1996; Myrskylä et al. 2009; Bray et al. 2012; Chang et al. 2013). The index and its underlying dimension indices are widely used in academic and policy-making circles, as well as in the broader community interested in development issues. The simplicity of the index's characterization of development, linked to the basic message that development is about more than economic growth, has contributed to its popularity (Klugman et al. 2011).

During the past decades, the living conditions of humankind-as indicated by the HDI and its underlying indices-have improved considerably (see the UNDP Human Development Reports (HDR) from 1990 onwards). On average, human beings now live longer (Riley 2001), are better educated (Morrisson and Murtin 2009), and enjoy a better standard of living (Easterlin 2000). Yet, whenever these general improvements in human development are shared inequitably and benefit some groups to the detriment of others, it is difficult to speak about unequivocal social progress (Rawls 1971; Sen 1999; HDR 2019).

Attempts to precisely measure the distribution of human development around the world face an important limitation: The HDI and its dimension indices are reported at the country level, and relatively little is known about the way they are distributed within countries. While there are clear indications that subnational variation in human development can be substantial (Foster et al. 2005; Grimm et al. 2008, 2010; Harttgen and Klasen 2011a, 201 1b; Permanyer 2013; Permanyer et al. 2015), until now it was not possible to study these differences for more than a handful of countries. Here, we document, for the first time, the levels and trends in within and betweencountry variation in human development across the globe.

To do this, we take advantage of the recently developed Subnational Human Development Database (SHDD) (Smits and Permanyer 2019), which provides a subnational version of the HDI (the so-called SHDI) and its three subcomponents for more than 1,600 regions within 161 countries, together covering over 99 percent of the world's population. This SHDI and its underlying indicators have at the national level the same values as the UNDP's HDI and its indicators, but show how the HDI and its indicators vary within countries.

Providing a 10-times higher resolution picture than was previously available, the new database opens the possibility of studying global socioeconomic variation and change with unprecedented coverage and detail and increases the ability of policy-makers to monitor and achieve the sustainable development goals. Here we use this SHD Database to report global trends in human development inequality since the year 2000, to investigate (i) how much of that inequality can be attributed to differences occurring between or within countries, (ii) which of the three components is mostly responsible for the observed levels of SHDI inequality within countries, and (iii) what the main drivers are of global inequality change since 

the turn of the twenty-first century. Unlike previous measures aimed at estimating "inequality-adjusted" levels of human development-like the "Inequality-adjusted HDI," or IHDI (see UNDP 2016)-here we focus on the discrepancy in the levels of human development across subnational regions around the world.

There exists already a huge literature on within- and between-country inequalities in income and nonincome dimensions of welfare. Many studies have investigated subnational variation in education (Jordá and Alonso 2016; Morrisson and Murtin 2013; Graetz et al. 2018), health (Smits and Monden 2009; Edwards 2011), or income (Anand and Segal 2015; Milanovic 2016; Bourguignon and Morrisson 2002; Nordhaus and Chen 2016) across the world or in specific regions. However, these studies tended to analyze these dimensions separately. The current study is different in that it documents global trends in education, health, and standard of living simultaneously - thus offering a unique opportunity to investigate how these factors have jointly evolved and influenced each other over time.

\section{Data}

The data used for this study are derived from the SHDD Database (Smits and Permanyer 2019). This database includes values of the HDI and of the underlying indicators and dimension indices for 1,625 regions in 161 countries. To construct the SHDI for these regions, the same kind of data and method was used as is used by the UNDP to construct their national HDI. The required data consist of four indicators: life expectancy at birth, mean years of schooling of adults $25+$, expected years of schooling of children aged 6, and gross national income per capita (GNIc, measured with purchasing power parity in 2011 US\$). The national values of these indicators were derived from the HDI database of the UNDP (http://hdr.undp.org/en/data). The subnational values are based on data obtained from statistical offices and from the Area Database of the Global Data Lab (GDL-AD), which includes subnational indicators constructed on the basis of household surveys (Smits 2016). For countries or years for which not all required indicators were available, estimations were based on related indicators (e.g., under5 mortality for life expectancy and household wealth as proxy for GNIc) or generated by using interpolation and extrapolation techniques (see the online Appendix containing supplementary materials for details). To create the best possible estimates given the data limitations, the subnational indicators were scaled in such a way that their national averages for a given year are equal to the national UNDP values for that year. This has the advantage that only the information on subnational variation had to be derived from statistical offices and survey datasets and that the constructed indicators, 

dimension indices, and SHDI index are at national level in line with their official UNDP values.

Further details of the approach used by Smits and Permanyer (2019) to construct the SHDI, including validity tests for the estimation procedures that were used, are presented in the online Appendix.

\section{Methods}

To compute the SHDI, we first estimate the education, health, and standard of living subcomponents $\left(E_{i}, H_{i}, S_{i}\right)$ and scale them between 0 and 1 using the same normalization procedures as the ones used in the official HDI (UNDP 2016). The education subindex is computed as ((EYS/18) $(M Y S / 15))^{1 / 2}$, where EYS and MYS are the expected and mean years of schooling, respectively. The health subindex is computed as $\left(e_{0}-20\right) /(85-$ $20)$, where $e_{0}$ is the life expectancy at birth. Third, the standard of living subindex is computed as $(\log ($ GNIc) $-\log (100)) /(\log (75000)-\log (100))$, where GNIc is the gross national income per capita (2011 purchasing power parity \$). When performing such normalization, we use the same goal posts for the entire period to facilitate comparisons over time. Mimicking the most recent definition of UNDP's HDI, the SHDI for each subnational area $i$ is defined as

$$
S H D I_{i}=\sqrt[3]{H_{i} E_{i} S_{i}} .
$$

This is the geometric mean of the three subcomponents (the use of the arithmetic mean to generate an additive SHDI does not alter the main findings of the paper). By taking the geometric mean, perfect substitutability between the health, education, and standard of living dimensions is prevented and regions are penalized for bad performance in one of the dimensions. Like the original HDI, the SHDI takes values between 0 and 1 (the former is reached whenever one of the three components attains the lowest possible level of 0 and the latter when all three components attain the maximal level of 1).

\section{Measuring and decomposing inequality}

To measure global inequality in the SHDI and its subcomponents, we have used two well-known inequality measures: the Gini index and the mean log deviation. The Gini is chosen for its popularity and the mean log deviation for its decomposability properties. The mean log deviation allows decomposing global SHDI inequality into within- and between-country components, and the Gini index can be used to assess how much of the SHDI inequality in a given country can be attributable to the education, health, or standard of living components. Lastly, we use a method inspired by the so-called "Shapley decomposition techniques" (Shorrocks 2013) that 

FIGURE 1 Global ventile distribution of the SHDI in 2017

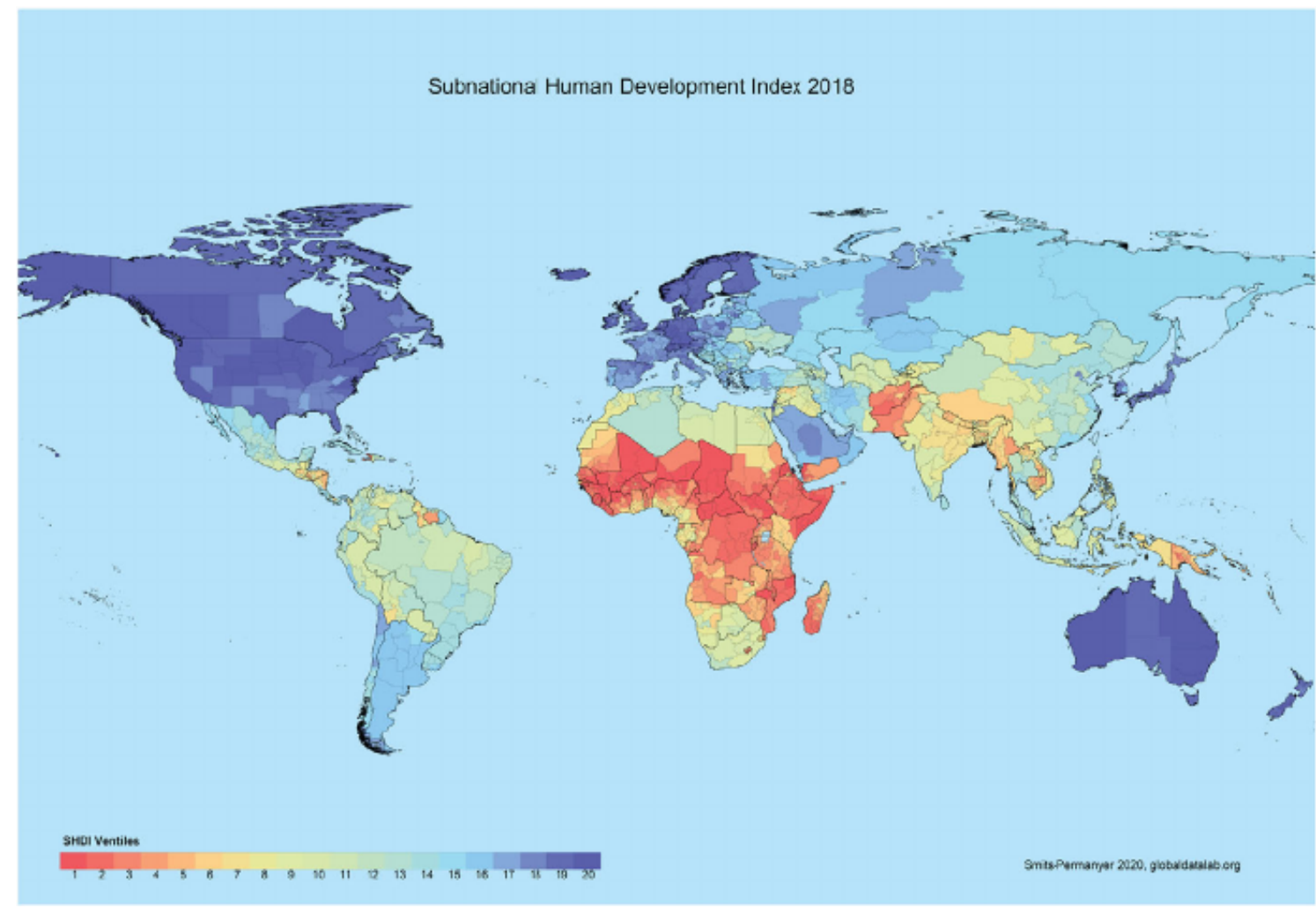

SOURCE: Authors' elaboration based on information from the Global Data Lab.

allows decomposing changes in global inequality $(\Delta I)$ into the sum of three clearly interpretable components: (i) changes in average attainment $\left(\Delta_{\mu} I\right)$, (ii) changes in within-country inequality $\left(\Delta_{T} I\right)$, and (iii) changes in population size $\left(\Delta_{P} I\right)$ - that is, $\Delta I=\Delta_{V} I+\Delta_{\mu} I+\Delta_{p} I$. Details and formal definitions are given in the online Appendix. While decomposition methods do not establish causation, they provide a "heuristically useful and methodologically transparent approach in which researchers can easily identify the main sources (if not causes) of change" (Eloundou-Enyegue et al. 2017, 60).

\section{Results}

Figure 1 shows the distribution of human development across the globe in 2017. We see that human development varies considerably not only among but also within countries. The finely grained scale of the SHDI allows identifying clusters of high-, middle-, and low-human development that cut across national borders (e.g., the highly developed regions surrounding the Alps, the middle-developed coastal regions bordering the Gulf of Guinea, or the least developed regions in landlocked sub-Saharan African countries).

In other cases, one can observe clear geographic patterns within countries (e.g., north-south divides in Belgium, Germany, Italy, and Spain). Some countries exhibit large regional variations (e.g., China, India, or Colombia) while others are quite homogeneous (e.g., Australia). Very 

FIGURE 2 Global inequality trends across $+1,600$ subnational regions (as measured with the Gini index) for the SHDI and its education, health, and standard of living subindices in years 2000, 2005, 2010, and 2015

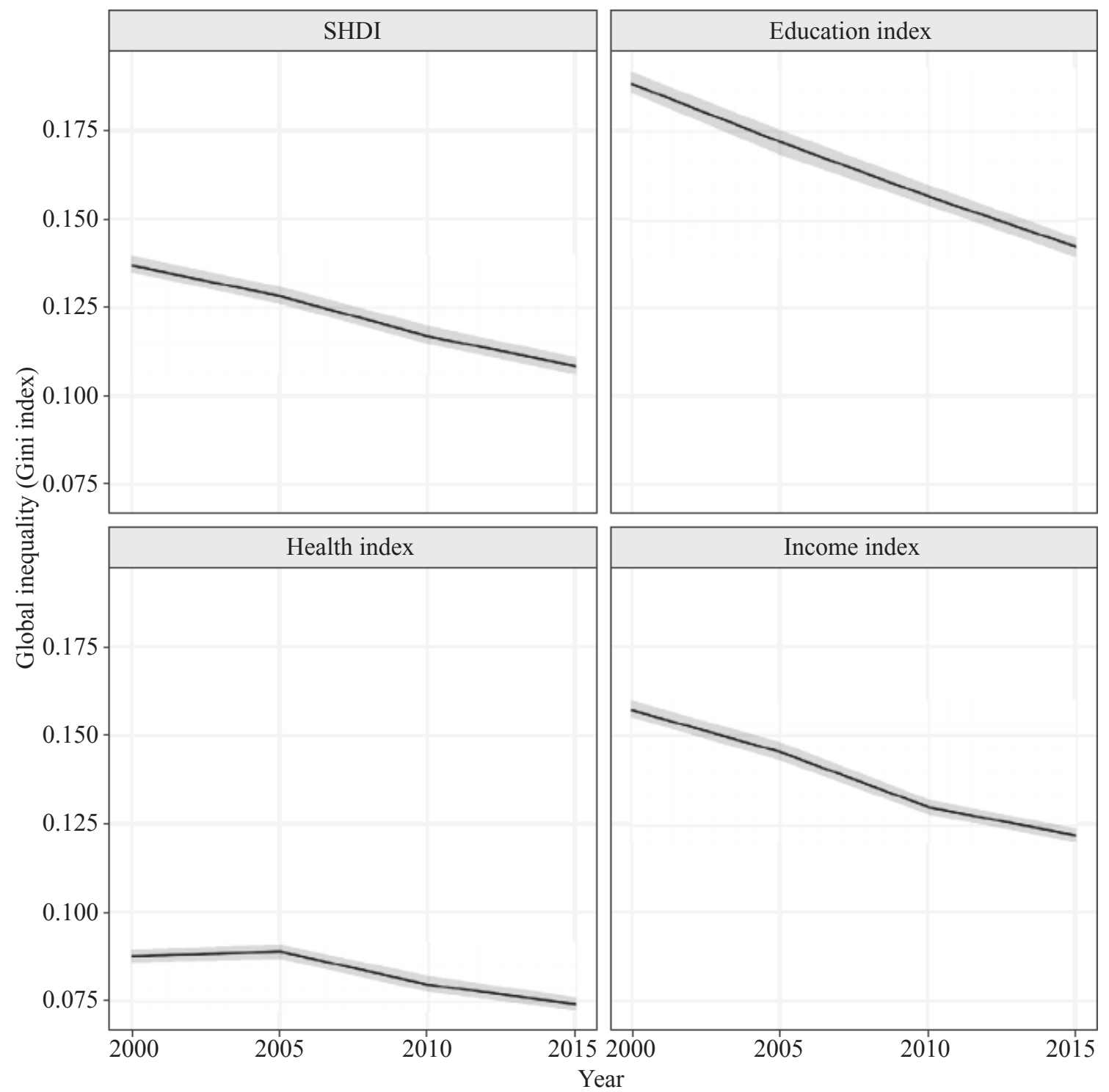

NOTE: The gray shaded areas show the size of 95 percent confidence intervals. SOURCE: Authors' elaboration based on information from the Global Data Lab.

often, the region where the capital city is located exhibits the highest human development levels and remote rural regions the lowest.

\section{Global inequality declines over time}

How has global inequality in SHDI (i.e., inequality across the 1,625 subnational regions for which the SHD database includes information) and its three components evolved since the turn of the century? In Figure 2, the Gini index for the global distribution of the SHDI and its three subcomponents is presented for the years 2000, 2005, 2010, and 2015. The Gini index is a broadly used inequality measure that runs from zero 

(complete equality) to one (maximum inequality) (Jenkins and Van Kerm 2011). Figure 2 shows that the inequality in the global SHDI distribution has monotonically decreased from 0.14 in 2000 to 0.11 fifteen years later. This reduction of inequality has taken place against a backdrop of general improvements in human development across and within countries.

Figure 2 also shows the trends in global inequality for the underlying health, education, and standard-of-living dimension indices. They are all decreasing, except for a small blip in 2005 for the health component-which might be attributable to the HIV/AIDS epidemic in sub-Saharan Africa and the aftermath of the Soviet Union's collapse (Goesling and Firebaugh 2004). However, we observe substantial differences in the magnitudes and speed of the decline. According to the Gini index, differences in the life expectancy index across world regions are smaller than differences in the education index. The former declined from 0.09 in 2000 to 0.08 in 2015, whereas the latter went from 0.19 to 0.14 in the same period. Somewhere in between, inequality in the standard-of-living index went from 0.16 to 0.12 . As the more unequal indices showed a faster decline, convergence is taking place towards the more equally distributed component (health).

In Figure 2, we also show the 95 percent confidence intervals resulting from a sensitivity analysis exercise aiming to assess the effect of measurement errors on global inequality trends (see the online Appendix for details). As can be seen, the uncertainty around the estimation of the SHDI distribution does not overly affect our results. The 95 percent confidence intervals are very narrow, both for the SHDI and for its different subcomponents, so the reported trends in global inequality are robust to the presence of measurement errors.

\section{Inequality within and between countries}

One of the most interesting features of the SHDI and its subcomponents is that we can quantify the amount of inequality that can be attributed to differences occurring within and between countries. In Figure 3, we show the percentages of total inequality that can be attributed to within-country differences in the world as a whole and in the groups of "low-," "medium," and "high-" HDI countries in 2015 (the results for 2000 are available in the online Appendix Figure A1). These percentages can be seen as the amount of "new" inequality that is revealed by looking beyond countrylevel averages using a subnational index.

Most striking in Figure 3 is the very high contribution of withincountry inequality to total inequality in the groups of countries at low- and intermediate levels of development (where as much as 70 percent of the world population lives). In these groups of countries, about half of inequality in SHDI is within-country inequality. Stated otherwise: when switching from national HDI to subnational SHDI, the amount of inequality we 

FIGURE 3 Percent contribution of within country inequality to total inequality for the SHDI and its three subcomponents in different groups of countries (year 2015)

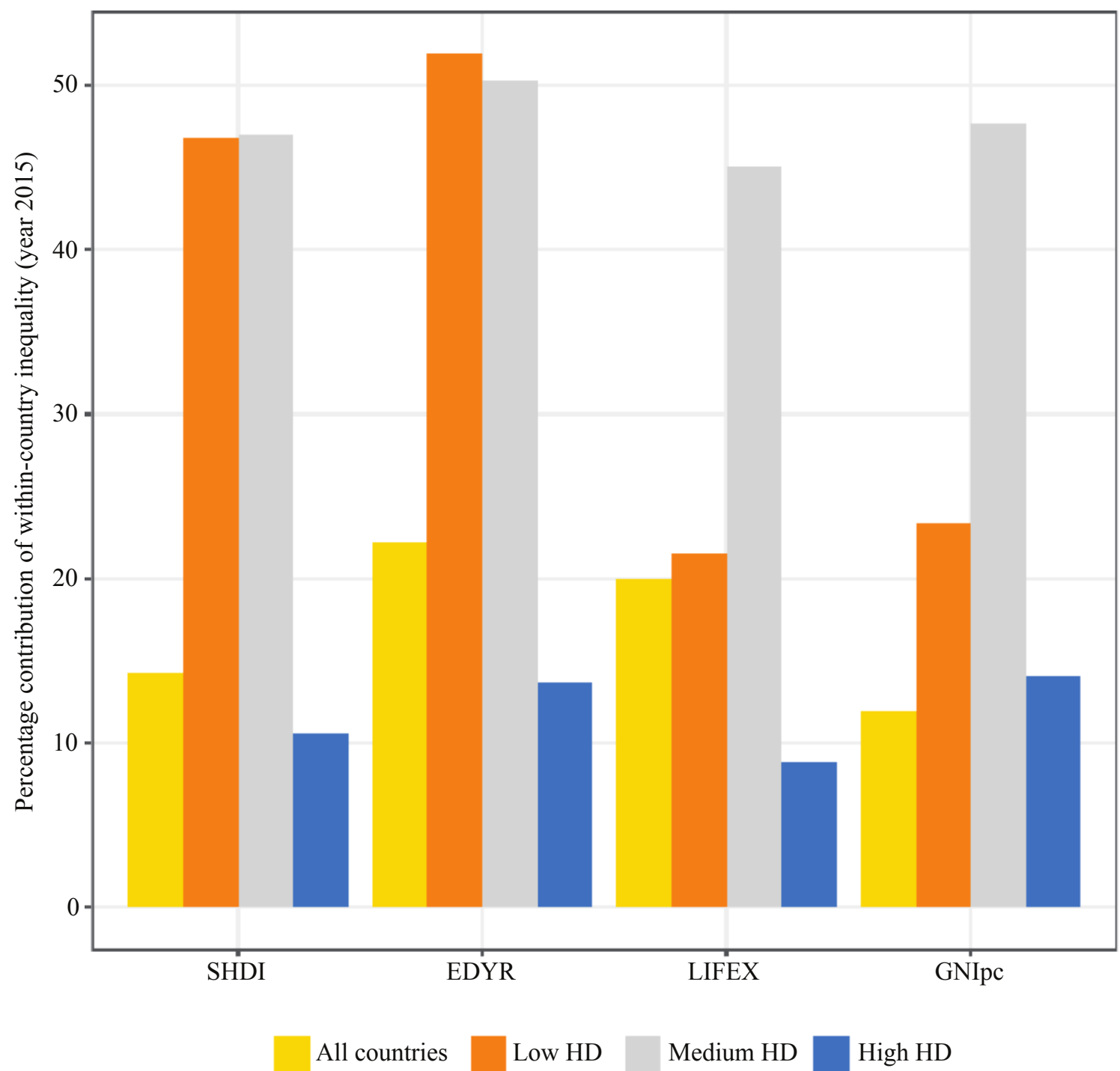

NOTE: To measure the within-country contribution we have used the Mean Log Deviation as inequality measure (which is additively decomposable and is very highly correlated with the values of the Gini index - see Supplementary Information section). To define whether a country belongs to the group of "Low-", "Medium-" or "High-" HDI countries we look at its HDI in year 2008 (the middle of the observation period). If such HDI is below (resp. above) 0.55 (resp. 0.7) the country belongs to the set of Low (resp. High) developed countries. Whenever it falls between 0.55 and 0.7 , it is a Medium developed country. The shares of the world population in Low-, Medium- and High developed countries are $20 \%, 50 \%$ and $30 \%$, respectively. The $50 \%$ horizontal line indicates the threshold above which the within-country component of inequality contributes more to total inequality than the between-country one.

SOURCE: Authors' elaboration based on information from the Global Data Lab.

observe in the regions of low- and middle-HDI countries doubles. Among the high-developed countries, within-country inequality is less important and most SHDI inequality is explained by differences between countries.

A rather similar pattern is observed when inspecting the three subcomponents of the SHDI separately (see Figure 3). Once again, the percentage of total inequality in education, health, and standard of living that can be attributed to within-country inequality is relatively small for 

the group of high-HDI countries (contributions hovering around 10 and 15 percent), but is much larger-and generally increasing-for low- and, particularly, for medium-HDI countries (with contributions often approaching or even above 50 percent). In those groups of countries, the newly found within-country differences in education, health, and standard of living carry about the same weight as the (already well-investigated) differences between countries.

\section{Explaining within country inequality}

Moving forward, one might wonder how much of the newly discovered HDI inequality within countries can be attributed to differences in education, health, and standard of living. Figure 4 plots for the groups of low-, medium-, and high-HDI countries, the contribution of the health $(H)$, education $(E)$, and standard of living $(S)$ subcomponents to SHDI inequality within countries (the online Appendix section explains how to perform such decompositions). The upper-, middle-, and lower panels show the results. Each dot in these ternary plots represents the three contributions for a specific country-year combination. The closer a dot is to a given vertex, the more important is the corresponding subcomponent $(H, E$, or $S)$ in explaining SHDI inequality within countries.

In the least developed countries, we observe that most observations (69 percent) are located near the education vertex $(E)$, while only 19 and 12 percent of them have standard of living $(S)$ and health $(H)$ as its closest vertex, respectively. This means that in those countries, within-country SHDI inequality is mostly due to variation in education. At the intermediate level of development, education is still most important in explaining within-country variation, but the influence of standard of living is growing ( 52 percent of points have $E$ as its closest vertex, and 33 percent of points are closest to the $S$ vertex). In the high developed countries, standard of living surpasses education as the most important explanatory factor for within country SHDI variation (50 and 45 percent of the points have $S$ and $E$ as their nearest vertex in the lower panel, respectively). For all three groups of countries, differences in the health component tend to be least important. Its influence is relatively strongest at the intermediate level of development (with 15 percent of points nearest to $\mathrm{H}$ ) and weakest in the most developed countries (with only 5 percent nearest to $\mathrm{H}$ ).

\section{What is driving global inequality trends?}

The level of global inequality $(I)$ is a function of the following elements: (1) countries' average attainment $(\mu),(2)$ within-country variation $(V)$, and (3) countries' population size $(p)$. Depending on the rate of change of these three elements, global inequality will increase or decrease over time. 

FIGURE 4 Contribution of education $(E)$, health $(H)$, and standard of living $(S)$ dimensions to SHDI inequality within countries by level of development
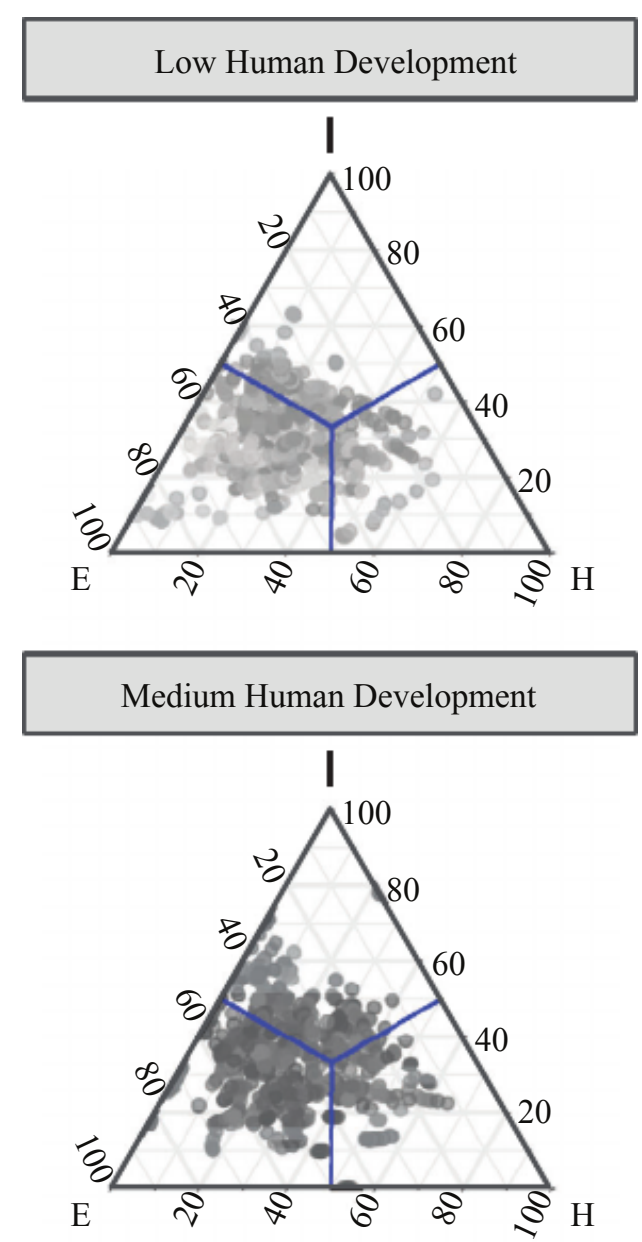

High Human Development

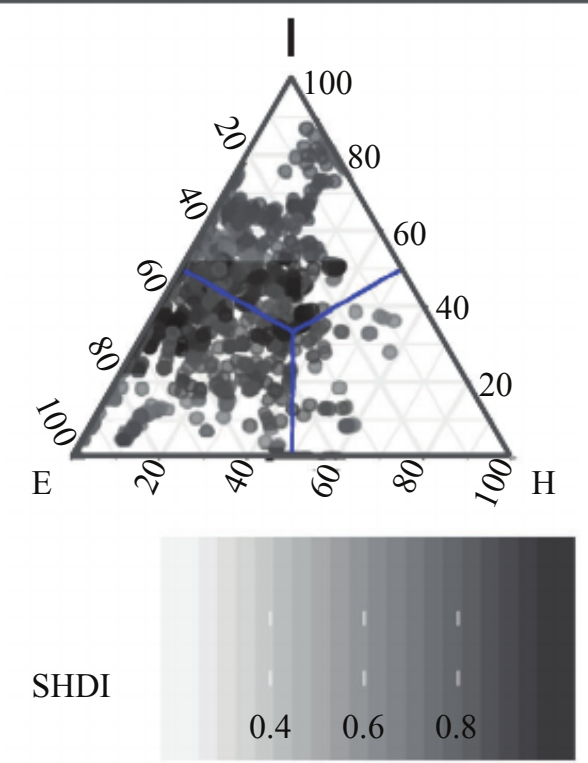

NOTE: The upper, middle and lower panels show the contributions for the groups of Low-, Medium- and High- HDI countries, respectively. Explanations of how to estimate the contribution of each indicator are given in the Supplementary Materials. Ternary plots show the contribution of the three components to SHDI inequality within each country: the position of each dot with respect to the vertices helps understanding how important the education, health and standard of living subcomponents are. The closer a dot is to a given vertex (say, E, H or S), the more important the corresponding subcomponent is. A hypothetical point in the middle of the triangle would represent a case where the three subcomponents contributed equally to observed SHDI inequality in a specific country. Another point near the top of the triangle would represent a case where most of the SHDI inequality would be explained by the standard of living component. The numbers inside the triangles indicate the percentage of observations that are closest to the $\mathrm{E}, \mathrm{H}$ or $\mathrm{S}$ vertices. 

Relying on a decomposition method, we report the impact that these three components have had on global inequality trends for the SHDI and its education, health, and standard of living subindicators. This method allows writing changes in inequality between two points, $t_{1}$ and $t_{2}(\Delta I)$, as the addition of three components, one reflecting the effect of changing average attainments $\left(\Delta_{\mu} I\right)$, another reflecting the effect of changing withincountry inequality $\left(\Delta_{V} I\right)$, and the third reflecting the effect of changing population sizes $\left(\Delta_{p} I\right)$ (details shown in the online Appendix). These effects will be referred to as "growth," "distribution," and "population" effects, respectively.

The first row in Table 1 shows how the 0.0133 units' decline in global SHDI inequality (from 0.031 in 2000 to 0.0178 in 2017 according to the mean $\log$ deviation $L$ ) can be decomposed as $-0.0156-0.0014+0.0038$, where the three quantities indicate the change in global SHDI inequality that is attributable to the growth, distribution, and population effects, respectively. The negative values associated with the first two effects mean that the evolution of countries' average attainment and, to a lesser extent, within-country variability has favored a decrease in global SHDI inequality, while the positive value associated with the third one indicates that the evolution of countries' population size has pushed it upwards. The fact that population changes tend to increase global SHDI inequality is a result of the negative bivariate relationship between 2000 SHDI levels and 2000-2017 population growth. Other factors kept constant, if an increasing share of the world population is living in countries at the lower levels of development, global inequality will increase. The large contribution of the growth effects to the reduction of SHDI inequality (75 percent; see last three columns in Table 1) indicates that countries at the lower tail of the HDI distribution have improved at a quicker pace than their more highly developed counterparts. This strong equalizing force has more than compensated for the (inequality-enhancing) population growth of low-HDI countries.

When the same decomposition exercise is performed for the groups of countries with "low," "medium," and "high" human development levels separately, different patterns arise (see rows 2, 3, and 4 in Table 1). For the groups of low- and middle-HDI countries (rows 2 and 3), distribution effects account for about one third of the declines in SHDI inequality (contributions around 30 percent). Thus, an important part of the success of the developing world in reducing inequality in human development between 2000 and 2017 is attributable to reductions in within-country inequality.

The other rows in Table 1 show the inequality change decomposition results for the education, health, and standard-of-living indicators of the SHDI separately. In general, the results differ in magnitude, but they roughly go in the same direction. Population growth tends to boost inequality, but this force is offset by growth effects and distribution-related factors which 



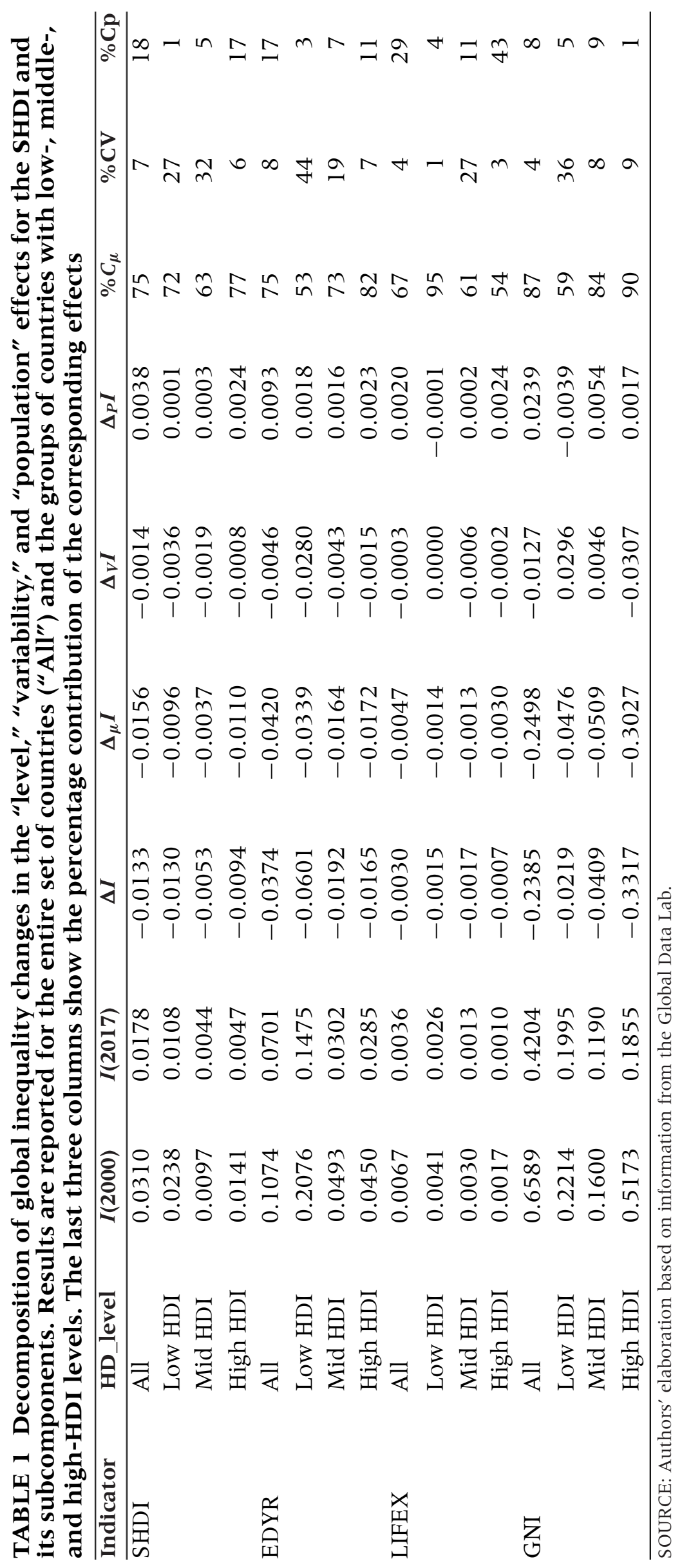



are inequality -depressing. An interesting exception to this trend can be seen in the standard of living component for low- and middle-HDI countries (see bottom panel in Table 1). For those groups of countries, changes in within-country variability have contributed to increase inequality rather than decreasing it.

\section{Sensitivity analysis}

Previous studies investigating trends in global inequality have explored the implications of dropping specific countries or regions from the analysis to find out to what extent overall trends are driven by the behavior of highly populated areas (e.g., Goesling and Firebaugh 2004; Chen and Ravallion 2010; Anand and Segal 2015; Milanovic 2016). Results of these studies suggest that particularly China has contributed considerably to the reductions in global income inequality and poverty in recent decades. In this section, we repeat our analysis of global inequality reported in Figure 2, but with the exclusion of (i) China, (ii) India, and (iii) sub-Saharan Africa from the analysis. We have chosen China and India because of their large population size (put together they account for around 40 percent of the world population) and sub-Saharan Africa because it is the region of the world that tends to fare worst in many socioeconomic indicators.

The findings are reported in Figure 5, where the thick continuous lines show the same global inequality trends reported in Figure 2, both for the SHDI and its three subindices. Excluding China from the analysis invariably increases the levels in global inequality for the SHDI and its subcomponents (e.g., in 2000, the Gini index of the SHDI distribution moves from 0.14 to slightly above 0.15 when removing that single country). Thus, as found in earlier studies, China can be seen as an equalizer of the global distribution of human development. Yet, the global inequality trends remain unaffected: even without China, the world converges towards equality across all indicators.

When repeating the same exercise for the case of India, we find that the overall levels and trends are largely unaffected: global inequality in human development without India is very similar to the inequality we observe when all subnational regions are included. This suggests that the human development levels of the Indian regions are distributed near the world's mean between 2000 and 2015 .

Lastly, dropping sub-Saharan Africa from the analysis reduces the extent of global inequality for the SHDI and its subcomponents. Being at the lower tail of the distribution, sub-Saharan regions thus contribute to increase global inequality in human development. Inspecting trends, we see that the world without sub-Saharan Africa would converge towards equality at a substantially faster pace than what is actually observed, both for the SHDI and for all its subcomponents. 

FIGURE 5 Global inequality trends across all $(+1,600)$ subnational regions and for selected groups of regions; for the SHDI and its education, health, and standard of living subindices in years 2000, 2005, 2010, and 2015

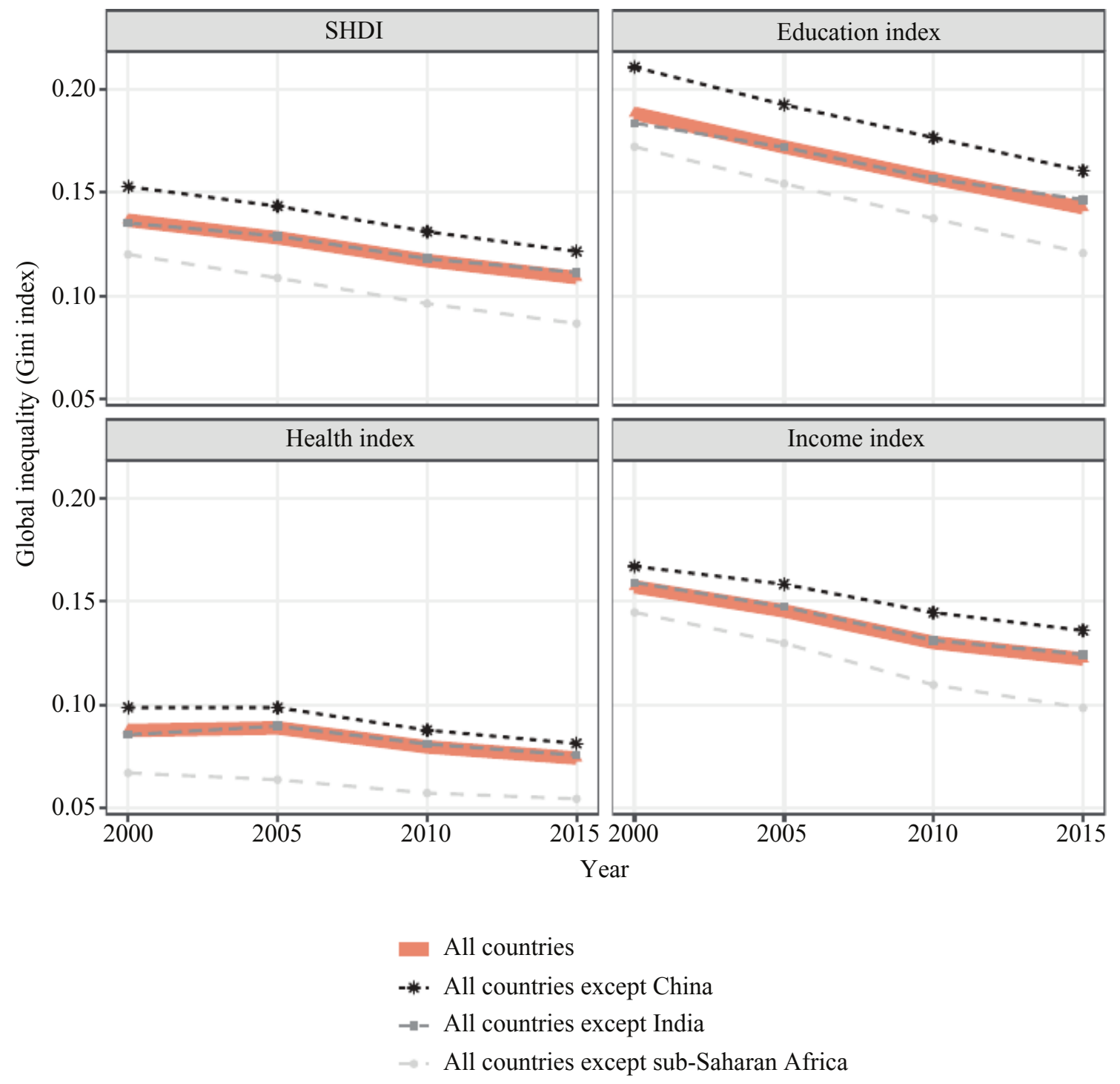

SOURCE: Authors' elaboration based on information from the Global Data Lab.

\section{Discussion}

By introducing subnational variation in HDI, we obtain a richer and more comprehensive picture of the distribution of human development across the globe. The amount of inequality that is measured increases considerably when subnational variation is included in the picture. In low- and middle-HDI countries (home to 70 percent of the global population), total inequality almost doubles. This result makes clear that national-level aggregates might conceal huge within-country inequalities in human development. For the education, health, and standard of living subindices and their underlying indicators, a similar picture is obtained. 

Within-country inequality in human development and subindices is much less in high-HDI countries, where it amounts to 10-15 percent of total inequality. These countries have more resources at hand to establish reasonable education and health facilities in their more remote rural areas. For low- and middle-HDI countries, this is much more difficult to achieve. With their restricted resources, they generally can only afford good facilities in the capital and major urban areas and access to these facilities is often only possible for the wealthier part of their population.

In low-HDI countries, within-country inequality in human development is to a large extent (two-thirds) the result of inequality in the education dimension. At the middle level of human development, within-country inequality in education is still the most important contributing factor, explaining about half of inequality, but standard of living is becoming more important, with a contribution of one-third. At the highest levels of development, standard of living has surpassed education as the most important contributor to within-country inequality in human development, explaining about 50 percent against 45 percent for education. At all three levels, difference in health contribute relatively little to within-country inequality in human development.

Our trend analysis revealed that in the period 2000-2015 global inequality in human development and its subdimensions decreased. At the same time, we observed that the importance of within country-inequality has increased, in particular at the lower and middle levels of development. The global decrease in human development inequality is to a large extent (75 percent) due to an increase in the average level of human development across the globe, whereby countries at the lower level of development have increased their human development at a faster pace than countries at the higher levels of development. It is to a small extent ( 7 percent) also the result of a reduction of within-country inequality, which implies that subnational regions with lower levels of development have improved at a somewhat larger speed than regions with higher levels of development. A more substantial (18 percent) contribution to the change, but in opposite direction (increasing global inequality), can be attributed to changes in population size of countries, whereby countries with lower development levels have seen their population growing at a faster pace than countries with higher development levels. For the subindices and the different levels of development separately, similar patterns can be observed, with growth effect by far contributing mostly to inequality reduction and population effects generally working in the opposite direction.

Some limitations of this study should be highlighted. First, like any composite index, both the HDI and its subnational version discussed here are restricted in their scope. Although of great importance, the three dimensions included in the (S)HDI are among other dimensions of well-being (e.g., environmental sustainability, gender equality, political freedom; Sagar 

and Najam 1998). Also the way in which the information is aggregated (i.e., the choice of dimensional weights and aggregation functions) could be chosen differently (Anand and Sen 1992; Neumayer 2001; Ranis et al. 2007; Cherchye et al. 2008; Foster et al. 2013; Permanyer 2012; Ravallion 2012). Despite these restrictions, the HDI plays a fundamental role in raising awareness that "development is much more than economic growth" (Klugman et al. 2011).

Second, several data points in the online SHDI Database have been estimated using different techniques. Missing data points have been filled using interpolation and extrapolation techniques, and missing subnational observations for the health and standard of living components have been estimated on the basis of under- 5 mortality rates and the International Wealth Index, respectively (details are given in the online Appendix). Analyses shown in the online Appendix demonstrate that the measurement error that entails from such estimation techniques is restricted. The estimated relative errors for inter- and extrapolation are quite small and the fit of the models to estimate the standard of living and health components is very good (with adjusted $R^{2}$ of 0.83 and 0.89 , respectively). Hence, while measurement errors could somewhat affect the SHDI estimates for specific subnational regions, it is unlikely that they would distort the overall trends reported in this article.

Third, the results presented in this paper uncover subnational variation in human development at the level of the main administrative units of each country. With on average about 10 regions per country, this is a great step forwards compared to the national indices used until now. However, given that the regions generally are rather large, part of inequality in SHDI might still remain concealed. Our figures therefore only provide a lower bound to SHDI inequality, and total global inequality is likely to be higher. Some exploratory analysis performed for the cases of Mexico (Permanyer 2013) and Brazil (UNDP Brazil 2013) with a municipal-level HDI suggest that variations at lower levels of aggregation can be potentially large. However, given that greater geographical detail (e.g., municipalities (Permanyer 2013) or small-scale grids (Graetz et al. 2018; Nordhaus and Chen 2016)) is only available for a restricted number of countries, this can currently only be obtained at the cost of losing comparability and missing the global picture. In addition, concepts like regional life expectancy, educational performance or standard of living can lose their meaning if the region becomes too small.

Despite such limitations, the database reported on in this article, and the analyses presented, significantly contributes to our understanding of inequality. The data on the SHDI and its components are freely accessible at https://hdi.globaldatalab.org/. This provides researchers worldwide with high-detail contextual variables that can be used to improve our understanding in wide-ranging areas of the social sciences (including, but not limited to, the study of family formation and fertility behavior, migration, 

health and mortality, epidemiology, cultural/ideational/normative change, religion, socioeconomic change, or environmental sustainability).

The patterns here described based on the SHDI are opposite to the long-established trends of ever-expanding between-country inequality that date back as far as the early stages of the Industrial Revolution. The shifting composition of human development inequality across and within countries implies that national location-while still of great importance-is losing prominence as a determining factor of individuals' well-being. The far-reaching implications of this change will shape the twenty-first century agenda of social scientists and policy-makers concerned with global distributive justice.

\section{Data availability statement}

The data used in this paper comes from the Subnational Human Development Database (SHDD), which can be freely and publicly accessed from the Global Data Lab (https://globaldatalab.org/shdi/) and from https://www. nature.com/articles/sdata201938.

\section{Conflict of interest}

The authors declare no conflict of interest.

\section{Note}

This work was supported by the European Research Council under Grant 637768; the Spanish Ministry of Economy and Competitiveness "Ramón y Cajal" Research Grant Program under Grant RYC-2013-14196; and the Spanish Ministry of Economy and Competitiveness National R\&D\&I Plan under Grant RTI2018-096730-B-I00. Different versions of this paper have been presented at different workshops and seminars (e.g., at UNDP's Human Development Report Office, the Global Family Change (GFC) project meetings and the 2019 ECINEQ meeting). We are grateful to their participants for their valuable comments and suggestions. In particular, we are indebted to Selim Jahan (HDRO-UNDP), Pedro Conceição (HDROUNDP), Milorad Kovacevic (HDRO-UNDP), Heriberto Tapia (HDRO-UNDP), Hans-Peter Kohler (University of Pennsylvania), and Frank Furstenberg (University of Pennsylvania).

\section{References}

Anand, Sudhir, and Amartya Sen. 1992. “Human Development Index: Methodology and Measurement." Human Development Report Office Occasional Paper 12. UNDP, New York.

Anand, Sudhir, and Paul Segal. 2015. "The Global Distribution of Income." In Handbook of Income Distribution, Volume 2A, edited by Anthony Atkinson and François Bourguignon, Chapter 11. Amsterdam: North Holland.

Bongaarts, John, and Susan C. Watkins. 1996. "Social Interactions and Contemporary Fertility Transitions." Population and Development Review 22(4): 639-682.

Bourguignon, François, and Christian Morrisson. 2002. "Inequality among World Citizens: 18201992." American Economic Review 92(4): 727-744. 

Bray, Freddie, Ahmedin Jemal, Nathan Grey, Jacques Ferlay, and David Forman. 2012. “Global Cancer Transitions According to the Human Development Index (2008-2030): A PopulationBased Study." The Lancet Oncology 13(8): 790-801.

Chang, Hannah, Jim Larson, Hannah Blencowe, Catherine Y. Spong, Christopher P. Howson, Sarah Cairns-Smith, Eve M. Lackritz., et al. 2013. “Preventing Preterm Births: Analysis of Trends and Potential Reductions with Interventions in 39 Countries with Very High Human Development Index." The Lancet 381 (9862): 223-234.

Chen, Shaohua, and Martin Ravallion. 2010. “The Developing World Is Poorer Than We Thought, But No Less Successful in the Fight against Poverty." The Quarterly Journal of Economics 125(4):1577-1625.

Cherchye, Laurens, Erwin Ooghe, and Tom Van Puyenbroeck. 2008. "Robust Human Development Rankings." Journal of Economic Inequality 6(4): 287-321.

Easterlin, Richard. 2000. "The Worldwide Standard of Living Since 1800." Journal of Economic Perspectives 14(1): 7-26.

Edwards, Ryan. 2011. "Changes in World Inequality in Length of Life: 1970-2000." Population and Development Review 37(3): 499-528.

Eloundou-Enyegue, Parfait, Sarah Giroux, and Michel Tenikue. 2017. “African Transitions and Fertility Inequality: A Demographic Kuznets Hypothesis." Population and Development Review 43(Issue S1): 59-83.

Foster, James, Luis Lopez-Calva, and Miguel Szekely. 2005. "Measuring the Distribution of Human Development: Methodology and an Application to Mexico." Journal of Human Development 6(1): 5-25.

Foster, James, Mark McGillivray, and Suman Seth. 2013. “Composite Indices: Rank Robustness, Statistical Association, and Redundancy." Econometric Reviews 32(1): 35-56.

Goesling, Brian, and Glenn Firebaugh. 2004. "The Trend in International Health Inequality." Population and Development Review 30(1): 131-146.

Graetz, Nicholas, Joseph Friedman, Aaron Osgood-Zimmerman, Roy Burstein, Molly H. Biehl, Chloe Shields, Jonathan F. Mosser, et al. 2018. "Mapping Local Variation in Educational Attainment across Africa." Nature 555: 48-53.

Grimm, Michael, Kenneth Harttgen, Stephan Klasen, and Mark Misselhorn. 2008. “A Human Development Index by Income Groups." World Development 36(12): 2527-2546.

Grimm, Michael, Kenneth Harttgen, Stephan Klasen, Mark Misselhorn, Teresa Munzi, and Timothy Smeeding. 2010. "Inequality in Human Development: An Empirical Assessment of 32 Countries." Social Indicators Research 97: 191-211.

Harttgen, Kenneth, and Stephan Klasen. 2011 a. "A Human Development Index by Internal Migration Status." Journal of Human Development and Capabilities 12(3): 393-424.

Harttgen, Kenneth, and Stephan Klasen. 201 1b. "Household-Based Human Development Index." World Development 40(5): 878-899.

Jenkins, Stephen, and Philippe Van Kerm. 2011. "The Measurement of Economic Inequality." In The Oxford Handbook of Economic Inequality, edited by Nolan, Brian, Wiemer Salverda, and Timothy Smeeding. Oxford, UK: Oxford University Press.

Jordá, Vanesa, and José M. Alonso. 2016. “New Estimates on Educational Attainment Using a Continuous Approach (1970-2010)." World Development 90: 281-293.

Klugman, Jeni, Francisco Rodríguez, and Hyung-Jin Choi. 2011. “The HDI 2010: New Controversies, Old Critiques." Journal of Economic Inequality 9(2): 249-288.

Milanovic, Branko. 2016. Global Inequality. A New Approach for the Age of Globalization. Cambridge, MA: Harvard University Press.

Morrisson, Christian, and Fabrice Murtin. 2009. "The Century of Education." Journal of Human Capital 3: 1-42.

Morrisson, Christian, and Fabrice Murtin. 2013. “The Kuznets Curve of Human Capital Inequality: 1870-2010." The Journal of Economic Inequality 11(3): 283-301.

Myrskylä, Mikko, Hans-Peter Kohler, and Francesco Billari. 2009. “Advances in Development Reverse Fertility Declines." Nature 460: 741-743. 

Neumayer, Eric. 2001. “The Human Development Index and Sustainability-A Constructive Proposal." Ecological Economics 39(1): 101-114.

Nordhaus, W. D., and Chen, X. 2016. “Global Gridded Geographically Based Economic Data (G-Econ), Version 4." Palisades, NY: NASA Socioeconomic Data and Applications Center (SEDAC). http: //doi.org/10.7927/H42V2D1C.

Permanyer, Iñaki. 2013. “Using Census Data to Explore the Spatial Distribution of Human Development." World Development 46: 1-13.

Permanyer, Iñaki. 2012. "Uncertainty and Robustness in Composite Indices Rankings." Oxford Economic Papers 64(1): 57-79.

Permanyer, Iñaki, Joan García, Albert Esteve, and Robert McCaa. 2015. "Human Development Index-like Small Area Estimates for Africa Compute from IPUMS-International Integrated Census Microdata." Journal of Human Development and Capabilities 16(2): 245271.

Ranis, Gustav, Frances Stewart, and Emma Samman. 2007. "Human Development: Beyond the Human Development Index." Journal of Human Development 7(3): 323-358.

Ravallion, Martin. 2012. "Troubling Tradeoffs in the Human Development Index." Journal of Development Economics 99(2): 201-209.

Rawls, John. 1971. A Theory of Justice. Cambridge, MA: The Belknap Press of Harvard University Press.

Riley, James. 2001. Rising Life Expectancy: A Global History. Cambridge, UK: Cambridge University Press.

Sagar, Ambuj, and Adil Najam. 1998. “The Human Development Index: A Critical Review." Ecological Economics 25: 249-264.

Sen, Amartya. 1999. Development as Freedom. New York: Oxford University Press.

Shorrocks, Anthony. 2013. "Decomposition Procedures for Distributional Analysis: A Unified Framework Based on the Shapley Value." Journal of Economic Inequality 11: 99-126.

Smits, Jeroen, and Iñaki Permanyer. 2019. "The Subnational Human Development Database." Scientific Data 6, 190038.

Smits, Jeroen, and Christiaan Monden. 2009. "Length of life inequality around the globe," Social Science $\theta$ Medicine 68: $1114-1123$.

Smits, Jeroen. 2016. "Sub-National Development Indicators for Research and Policy-Making." GDL Working paper 16-101.

UNDP (U.N. Development Programme)-Human Development Report Office. Human Development Reports 1990, 1991, 1993, 1995, 2009, 2010. New York: Oxford University Press 1990 through 2005; London: Palgrave Macmillan since 2006.

UNDP Brazil. 2013. Índice de Desenvolvimento Humano Municipal Brasileiro. Brasília: Ipea, FJP.

UNDP. 2016. Technical Notes. Human Development Report 2016. New York: U.N. Development Programme.

UNDP (2019), Human Development Report 2019: Beyond Income, Beyond Averages, Beyond Today - Inequalities in Human Development in the 21st Century, UN, New York, https://doi.org/10.18356/838f78fd-en. 
